\title{
EXISTENCE OF POSITIVE SOLUTIONS FOR NONLINEAR BOUNDARY VALUE PROBLEMS IN BOUNDED DOMAINS OF $\mathbb{R}^{n}$
}

\author{
FATEN TOUMI
}

Received 10 June 2004; Accepted 22 September 2004

Let $D$ be a bounded domain in $\mathbb{R}^{n}(n \geq 2)$. We consider the following nonlinear elliptic problem: $\Delta u=f(\cdot, u)$ in $D$ (in the sense of distributions), $u_{\mid \partial D}=\varphi$, where $\varphi$ is a nonnegative continuous function on $\partial D$ and $f$ is a nonnegative function satisfying some appropriate conditions related to some Kato class of functions $K(D)$. Our aim is to prove that the above problem has a continuous positive solution bounded below by a fixed harmonic function, which is continuous on $\bar{D}$. Next, we will be interested in the Dirichlet problem $\Delta u=-\rho(\cdot, u)$ in $D$ (in the sense of distributions), $u_{\mid \partial D}=0$, where $\rho$ is a nonnegative function satisfying some assumptions detailed below. Our approach is based on the Schauder fixed-point theorem.

Copyright (c) 2006 Faten Toumi. This is an open access article distributed under the Creative Commons Attribution License, which permits unrestricted use, distribution, and reproduction in any medium, provided the original work is properly cited.

\section{Introduction}

Let $D$ be a bounded $C^{1,1}$-domain in $\mathbb{R}^{n}(n \geq 2)$, and let $G$ be the Green function for the Laplace operator with zero Dirichlet boundary condition on $\partial D$. In [4], Chung and Zhao have established interesting inequalities for the Green function $G$. In particular, they showed that there exists a constant $C>0$ such that for each $x, y$ in $D$,

$$
\frac{1}{C} H(x, y) \leq G(x, y) \leq C H(x, y)
$$

where

$$
H(x, y):= \begin{cases}\frac{1}{|x-y|^{n-2}} \min \left(1, \frac{\delta(x) \delta(y)}{|x-y|^{2}}\right), & \text { if } n \geq 3, \\ \log \left(1+\frac{\delta(x) \delta(y)}{|x-y|^{2}}\right), & \text { if } n=2,\end{cases}
$$

and $\delta(x)$ denotes the Euclidean distance between $x$ and $\partial D$.

Hindawi Publishing Corporation

Abstract and Applied Analysis

Volume 2006, Article ID 95480, Pages 1-18

DOI 10.1155/AAA/2006/95480 
Another crucial inequality for the Green function $G$ called $3 G$-theorem is given by Kalton and Verbitsky [7] for $n \geq 3$ and by Selmi [12] for $n=2$, namely, there exists a constant $C_{0}>0$ depending only on $D$ such that for all $x, y$, and $z$ in $D$,

$$
\frac{G(x, z) G(z, y)}{G(x, y)} \leq C_{0}\left(\frac{\delta(z)}{\delta(x)} G(x, z)+\frac{\delta(z)}{\delta(y)} G(y, z)\right) .
$$

This 3G-theorem was investigated by Mâagli and Zribi [10], Zeddini [13], and Mâagli and Mâatoug [9] to introduce a new class of functions denoted by $K(D)$, (see Definition 1.1 below), which contains properly the classical Kato class introduced by Aizenman and Simon [1]. Moreover, they used the properties of functions belonging to this class $K(D)$ to study some nonlinear differential equations.

Definition 1.1. A Borel measurable function $q$ in $D$ belongs to the class $K(D)$ if $q$ satisfies

$$
\lim _{\alpha \rightarrow 0}\left(\sup _{x \in D} \int_{D \cap B(x, \alpha)} \frac{\delta(y)}{\delta(x)} G(x, y)|q(y)| d y\right)=0 .
$$

In this paper, we will exploit the properties pertaining to $K(D)$ to give some results about the existence of positive solutions of nonlinear elliptic problems. Our plan is as follows.

In Section 2, we establish some estimates on the Green function $G$ and some properties of functions belonging to the Kato class $K(D)$.

In Section 3, we are concerned with the existence of positive continuous solutions of the nonlinear elliptic problem

$$
\begin{array}{r}
\Delta u=f(\cdot, u) \quad \text { (in the sense of distributions), } \\
u>0 \quad \text { in } D, \quad u_{\mid \partial D}=\varphi
\end{array}
$$

where $\varphi$ is a nontrivial nonnegative continuous function on $\partial D$. Then, we fix a nontrivial nonnegative harmonic function $h_{0}$ in $D$, which is continuous in $\bar{D}$, and we suppose that $f$ satisfies the following hypotheses.

$\left(\mathrm{H}_{1}\right) f: D \times(0,+\infty) \rightarrow[0,+\infty)$ is measurable, continuous with respect to the second variable and satisfies

$$
f(x, t) \leq \theta(x, t), \quad \text { for }(x, t) \in D \times(0,+\infty),
$$

where $\theta$ is a nonnegative measurable function on $D \times(0,+\infty)$ such that the function $t \rightarrow \theta(x, t)$ is nonincreasing on $(0,+\infty)$.

$\left(\mathrm{H}_{2}\right)$ The function $\psi$ defined on $D$ by $\psi(x)=\theta\left(x, h_{0}(x)\right) / h_{0}(x)$ belongs to the class $K(D)$.

Remark 1.2. Note that the condition " $\forall c>0, \theta(\cdot, c \delta(\cdot)) / \delta(\cdot) \in K(D)$ " implies the hypothesis $\left(\mathrm{H}_{2}\right)$. Indeed, from [14], there exists $c>0$ such that for each $x \in D, h_{0}(x) \geq$ $c \delta(x)$. So, using the fact that $t \rightarrow \theta(x, t) / t$ is nonincreasing function on $(0,+\infty)$, we obtain $\left(\mathrm{H}_{2}\right)$. 
Under the assumptions $\left(\mathrm{H}_{1}\right)-\left(\mathrm{H}_{2}\right)$, we aim at proving the following result: there exists a constant $c>1$ such that if $\varphi \geq c h_{0}$ on $\partial D$, then problem (1.5) has a positive continuous solution $u$ satisfying for each $x \in D$,

$$
h_{0}(x) \leq u(x) \leq H_{D} \varphi(x)
$$

where $H_{D} \varphi$ is the harmonic continuous function having boundary value $\varphi$ on $\partial D$.

This result improves the one of Atherya [2], who considered the following problem:

$$
\Delta u=g(u) \quad \text { in } \Omega, \quad u_{\mid \partial \Omega}=\varphi,
$$

where $\Omega$ is a simply connected bounded $C^{2}$-domain in $\mathbb{R}^{n}(n \geq 3)$ and $g(u) \leq \max \left(1, u^{-\alpha}\right)$, for $0<\alpha<1$. He proved the existence of a positive continuous solution bounded below by a fixed positive harmonic function $h_{0}$ provided that there exists a positive constant $c>1$ such that $\varphi \geq c h_{0}$ on $\partial D$.

In the last section, we will study the following nonlinear problem:

$$
\Delta u=-\rho(\cdot, u) \quad \text { in } D \text { (in the sense of distributions), } \quad u_{\mid \partial D}=0,
$$

where $\rho$ is required to verify the following hypotheses.

$\left(\mathrm{H}_{3}\right) \rho$ is nonnegative Borel measurable function on $D \times(0, \infty)$, continuous with respect to the second variable.

$\left(\mathrm{H}_{4}\right)$ There exist $p, q: D \rightarrow(0, \infty)$ nontrivial Borel measurable functions and $h, k:(0$, $\infty) \rightarrow[0, \infty)$ nontrivial and nondecreasing Borel measurable functions satisfying

$$
p(x) h(t) \leq \rho(x, t) \leq q(x) k(t), \quad \text { for }(x, t) \in D \times(0, \infty),
$$

such that
$\left(\mathrm{A}_{1}\right) p \in L_{\text {loc }}^{1}(D)$,
$\left(\mathrm{A}_{2}\right) q \in K(D)$,
$\left(\mathrm{A}_{3}\right) \lim _{t \rightarrow 0^{+}}(h(t) / t)=+\infty$,
$\left(\mathrm{A}_{4}\right) \lim _{t \rightarrow+\infty}(k(t) / t)=0$.

Under these hypotheses, we will prove that (1.8) has a positive continuous solution $u$ satisfying on $D$,

$$
a \delta(x) \leq u(x) \leq b,
$$

where $a, b$ are positive constants.

Problem (1.8) has been studied by Dalmasso [5] on the unit ball with more restrictive conditions on $\rho$. Indeed, Dalmasso proved the existence of positive solutions provided that $\rho$ is nondecreasing with respect to the second variable and satisfies

$$
\lim _{t \rightarrow 0^{+}}\left(\min _{x \in \bar{B}} \frac{\rho(x, t)}{t}\right)=+\infty, \quad \lim _{t \rightarrow+\infty}\left(\max _{x \in \bar{B}} \frac{\rho(x, t)}{t}\right)=0 .
$$

When $\rho(x, t)=\rho(|x|, t)$, he showed the uniqueness of positive radial solution of (1.8). 
On the other hand, problem (1.8) has been studied on the entire space $\mathbb{R}^{n}$ by Brezis and Kamin [3] for the special nonlinearity $\rho(x, t)=\nu(x) t^{\alpha}, 0<\alpha<1$. More precisely they proved the existence and the uniqueness of positive solution for the problem below:

$$
\Delta u=-v(x) u^{\alpha} \quad \text { in } \mathbb{R}^{n} \quad \lim _{|x| \rightarrow \infty} \inf u=0
$$

Notations and preliminaries. In order to simplify our statement, we adopt the following notations.

(i) $C_{0}(D):=\left\{f \in C(D): \lim _{x \rightarrow \partial D} f(x)=0\right\}$.

We note that $C_{0}(D)$ is a Banach space endowed with the uniform norm

$$
\|f\|_{\infty}=\sup _{x \in D}|f(x)|
$$

(ii) Let $f$ and $g$ be two nonnegative functions on a set $S$.

We call $f \sim g$, if there exists a constant $c>0$ such that

$$
\frac{1}{c} g(x) \leq f(x) \leq c g(x) \quad \forall x \in S .
$$

We call $f \preceq g$, if there exists a constant $c>0$ such that

$$
f(x) \leq \operatorname{cg}(x) \quad \forall x \in S \text {. }
$$

(iii) Let $f$ be a nonnegative function in $D$, then we denote by $V f$ the potential of $f$ defined on $D$ by

$$
V f(x)=\int_{D} G(x, y) f(y) d y
$$

We recall that if $f \in L_{\text {loc }}^{1}(D)$ and $V f \in L_{\text {loc }}^{1}(D)$, then we have $\Delta(V f)=-f$ in $D$ (in the sense of distributions) (see [4, page 52]).

(iv) We denote by $d$ the diameter of $D$.

(v) For $x, y \in D$, we denote $[x, y]^{2}=|x-y|^{2}+\delta(x) \delta(y)$.

\section{Properties of the Green function and the class $K(D)$}

In this section, we establish some results concerning the Green function $G(x, y)$ and the Kato class $K(D)$.

Proposition 2.1 (see $[9,10]$ ). Let $q$ be a nonnegative function in $K(D)$. Then

(i) the potential $V q \in C_{0}(D)$,

(ii) the function $x \rightarrow \delta(x) q(x)$ is in $L^{1}(D)$. 
In the sequel, we put

$$
\begin{aligned}
\|q\|_{D} & =\sup _{x \in D} \int_{D} \frac{\delta(y)}{\delta(x)} G(x, y)|q(y)| d y, \\
\alpha_{q} & =\sup _{x, y \in D} \int_{D} \frac{G(x, z) G(z, y)}{G(x, y)}|q(z)| d z
\end{aligned}
$$

We recall that if $q \in K(D)$, then $\|q\|_{D}<\infty$.

Now, it is obvious to see that by (1.3), we have

$$
\alpha_{q} \leq 2 C_{0}\|q\|_{D}
$$

where $C_{0}$ is the constant given by (1.3).

Next, we will prove that $\alpha_{q} \sim\|q\|_{D}$.

Proposition 2.2. Let $q$ be a function in $K(D)$. Then

(i) for any nonnegative superharmonic function $h$ in $D$, we have

$$
\int_{D} G(x, y)|q(y)| h(y) d y \leq \alpha_{q} h(x), \quad \forall x \in D,
$$

(ii) there exists a constant $C>0$ such that

$$
C\|q\|_{D} \leq \alpha_{q}
$$

Proof. (i) Let $h$ be a nonnegative superharmonic function in $D$, then from $[11$, Theorem 2.1 , page 164$]$, there exists a sequence $\left(f_{k}\right)$ of nonnegative measurable functions on $D$ such that for all $y \in D$,

$$
h_{k}(y)=\int_{D} G(x, z) f_{k}(z) d z
$$

increases to $h(y)$.

Since for each $x, y \in D$, we have

$$
\int_{D} G(x, y)|q(y)| h_{k}(y) d y \leq \alpha_{q} h_{k}(x) .
$$

Thus, from the monotone convergence theorem, we deduce the result.

(ii) Let $\varphi_{1}$ be a positive eigenfunction corresponding to the first eigenvalue of the Dirichlet problem $\Delta u+\lambda u=0, u_{\mid \partial D}=0$. Then, from [8, Proposition 2.6] we have for $x \in D$

$$
\varphi_{1}(x) \sim \delta(x) .
$$

Since, $\varphi_{1}$ is a superharmonic function in $D$, then by applying (i) to $\varphi_{1}$, we deduce (ii).

Proposition 2.3. Let $p>n / 2$. Then for each $\lambda<2-n / p$, we have

$$
\frac{1}{(\delta(\cdot))^{\lambda}} L^{p}(D) \subset K(D) .
$$


To prove Proposition 2.3, we need the two next lemmas.

LeMma 2.4. On $D^{2}$, we have

(i) for $n \geq 3, G(x, y) \sim \delta(x) \delta(y) /|x-y|^{n-2}[x, y]^{2}$,

(ii) for $n=2, G(x, y) \sim\left(\delta(x) \delta(y) /[x, y]^{2}\right) \log \left(1+[x, y]^{2} /|x-y|^{2}\right)$.

Proof. (i) For each $a, b \geq 0$, we have

$$
\min (a, b) \sim \frac{a b}{a+b}
$$

So, by (1.1) we deduce (i).

(ii) Using (1.1), the fact that for each $t \geq 0, \log (1+t) \sim \min (1, t) \log (2+t)$, and $(2.10)$ we obtain that

$$
G(x, y) \sim \min \left(1, \frac{\delta(x) \delta(y)}{|x-y|^{2}}\right) \log \left(2+\frac{\delta(x) \delta(y)}{|x-y|^{2}}\right) \sim \frac{\delta(x) \delta(y)}{[x, y]^{2}} \log \left(1+\frac{[x, y]^{2}}{|x-y|^{2}}\right)
$$

Lemma 2.5. Let $\lambda \in \mathbb{R}$. Then on $D^{2}$, we have

$$
\frac{1}{(\delta(y))^{\lambda}} \frac{\delta(y)}{\delta(x)} G(x, y) \preceq \begin{cases}\frac{1}{|x-y|^{n-2+\lambda^{+}}}, & \text {if } n \geq 3, \\ \frac{1}{|x-y|^{\lambda^{+}}} \log \left(\frac{2 d}{|x-y|}\right), & \text { if } n=2,\end{cases}
$$

where $\lambda^{+}=\max (0, \lambda)$.

Proof. By Lemma 2.4, we have on $D^{2}$

$$
\frac{1}{(\delta(y))^{\lambda}} \frac{\delta(y)}{\delta(x)} G(x, y) \preceq \begin{cases}\frac{1}{|x-y|^{n-2}} \frac{(\delta(y))^{2-\lambda}}{[x, y]^{2}}, & \text { if } n \geq 3, \\ \frac{(\delta(y))^{2-\lambda}}{[x, y]^{2}} \log \left(1+\frac{[x, y]^{2}}{|x-y|^{2}}\right), & \text { if } n=2 .\end{cases}
$$

Now, we remark that

$$
[x, y]^{2} \sim|x-y|^{2}+4 \delta(x) \delta(y)
$$

So, we have

$$
\begin{aligned}
{[x, y]^{2} } & \succeq \max \left(|\delta(x)-\delta(y)|^{2}+4 \delta(x) \delta(y),|x-y|^{2}\right) \\
& \geq \max \left((\delta(y))^{2},|x-y|^{2}\right) .
\end{aligned}
$$


Therefore by (2.15) we have

$$
\frac{1}{[x, y]^{2}} \preceq \frac{1}{|x-y|^{\lambda^{+}}(\delta(y))^{2-\lambda^{+}}} .
$$

Hence, it follows that

$$
\frac{(\delta(y))^{2-\lambda}}{[x, y]^{2}} \preceq \frac{1}{|x-y|^{\lambda^{+}}}
$$

Thus, for $n \geq 3$, we obtain

$$
\frac{1}{(\delta(y))^{\lambda}} \frac{\delta(y)}{\delta(x)} G(x, y) \preceq \frac{1}{|x-y|^{n-2+\lambda^{+}}} .
$$

Next, it is obvious to see that

$$
\log \left(1+\frac{[x, y]^{2}}{|x-y|^{2}}\right) \leq \log \left(2 \frac{[x, y]^{2}}{|x-y|^{2}}\right) \leq \log \left(\frac{4 d^{2}}{|x-y|^{2}}\right) .
$$

Then, for $n=2$, we obtain by (2.17) and (2.19) that

$$
\frac{1}{(\delta(y))^{\lambda}} \frac{\delta(y)}{\delta(x)} G(x, y) \preceq \frac{1}{|x-y|^{\lambda^{+}}} \log \left(\frac{2 d}{|x-y|}\right) .
$$

This completes the proof.

Proof of Proposition 2.3. Let $\alpha>0, p>n / 2$ and $q \geq 1$ such that $(1 / p)+(1 / q)=1$. To show the claim, we use Lemma 2.5 and the Hölder inequality. We distinguish two cases.

Case $1(n \geq 3)$. Let $f \in L^{p}(D)$ and $\lambda<2-n / p$. Then, for $x \in D$, we have

$$
\begin{aligned}
& \int_{D \cap B(x, \alpha)} \frac{\delta(y)}{\delta(x)} G(x, y) \frac{|f(y)|}{(\delta(y))^{\lambda}} d y \\
& \quad \preceq \int_{D \cap B(x, \alpha)} \frac{|f(y)|}{|x-y|^{n-2+\lambda^{+}}} d y \preceq\|f\|_{p}\left(\int_{0}^{\alpha} r^{n(1-q)+\left(2-\lambda^{+}\right) q-1} d r\right)^{1 / q} \preceq\|f\|_{p} \alpha^{2-n / p-\lambda^{+}},
\end{aligned}
$$

which tends to zero as $\alpha \rightarrow 0$.

Case $2(n=2)$. Let $f \in L^{p}(D)$ and $\lambda<2 / q$. Then, for $x \in D$, we have

$$
\begin{aligned}
& \int_{D \cap B(x, \alpha)} \frac{\delta(y)}{\delta(x)} G(x, y) \frac{|f(y)|}{(\delta(y))^{\lambda}} d y \\
& \quad \preceq \int_{D \cap B(x, \alpha)} \frac{|f(y)|}{|x-y|^{\lambda^{+}}} \log \left(\frac{2 d}{|x-y|}\right) d y \preceq\|f\|_{p}\left(\int_{0}^{\alpha} r^{n-1-\lambda^{+} q}\left(\log \frac{2 d}{r}\right)^{q} d r\right)^{1 / q},
\end{aligned}
$$

which tends to zero as $\alpha \rightarrow 0$. This completes the proof. 
In the sequel, we put for $f \in \mathscr{B}(D)$ and $x \in D$,

$$
v(x)=\int_{D} G(x, y) \frac{|f(y)|}{(\delta(y))^{\lambda}} d y .
$$

Remark 2.6. From (1.1), we remark that for $x, y \in D$, we have $\delta(x) \delta(y) \preceq G(x, y)$. This implies that there exists a constant $C>0$ such that for each $f \in \mathscr{B}(D)$ and $x \in D$,

$$
C \delta(x) \int_{D}(\delta(y))^{1-\lambda}|f(y)| d y \leq v(x)
$$

In the next proposition, we will give upper estimates on the function $v$.

Proposition 2.7. Let $p>n / 2$ and $\lambda<2-n / p$. Then there exists a constant $c>0$, such that for each $f \in L^{p}(D)$ and $x \in D$,

$$
v(x) \leq \begin{cases}c\|f\|_{p}(\delta(x))^{2-n / p-\lambda}, & \text { if } 1-\frac{n}{p}<\lambda<2-\frac{n}{p}, \\ c\|f\|_{p} \delta(x)\left(\log \frac{2 d}{\delta(x)}\right)^{1 / q}, & \text { if } \lambda=1-\frac{n}{p}, \\ c\|f\|_{p} \delta(x), & \text { if } \lambda<1-\frac{n}{p} .\end{cases}
$$

To prove Proposition 2.7, we need the following lemma.

Lemma 2.8 (see [8]). Let $x, y \in D$. Then we have the following properties:

(i) if $\delta(x) \delta(y) \leq|x-y|^{2}$ then $\min (\delta(x), \delta(y)) \leq((\sqrt{5}+1) / 2)|x-y|$,

(ii) if $|x-y|^{2} \leq \delta(x) \delta(y)$ then $((3-\sqrt{5}) / 2) \delta(x) \leq \delta(y) \leq((3+\sqrt{5}) / 2) \delta(x)$.

Proof of Proposition 2.7. Let $p>n / 2, q \geq 1$ such that $(1 / p)+(1 / q)=1$ and $\lambda<2-n / p$. Let $f \in L^{p}(D)$, then for each $x \in D$, we have

$$
v(x)=\int_{D_{1}} G(x, y) \frac{|f(y)|}{(\delta(y))^{\lambda}} d y+\int_{D_{2}} G(x, y) \frac{|f(y)|}{(\delta(y))^{\lambda}} d y=I_{1}+I_{2},
$$

where

$$
\begin{aligned}
& D_{1}=\left\{y \in D: \delta(x) \delta(y) \geq|x-y|^{2}\right\}, \\
& D_{2}=\left\{y \in D: \delta(x) \delta(y) \leq|x-y|^{2}\right\} .
\end{aligned}
$$

Now, we remark that for each $x \in D$ and $y \in D_{1}$, we have by (1.1) and Lemma 2.8

$$
\frac{1}{(\delta(y))^{\lambda}} G(x, y) \preceq \begin{cases}\frac{1}{(\delta(x))^{\lambda}} \frac{1}{|x-y|^{n-2}}, & \text { for } n \geq 3, \\ \frac{1}{(\delta(x))^{\lambda}} \log \left(1+\left(\frac{2 \delta(x)}{|x-y|}\right)^{2}\right), & \text { for } n=2 .\end{cases}
$$


Then, by the Hölder inequality and Lemma 2.8, we obtain for $n \geq 3$

$$
\begin{aligned}
I_{1} & \preceq\|f\|_{p}(\delta(x))^{-\lambda}\left(\int_{D_{1}} \frac{1}{|x-y|^{(n-2) q}} d y\right)^{1 / q} \\
& \leq\|f\|_{p}(\delta(x))^{-\lambda}\left(\int_{0}^{((\sqrt{5}+1) / 2) \delta(x)} r^{n-1-(n-2) q} d r\right)^{1 / q} \\
& \leq\|f\|_{p}(\delta(x))^{2-\lambda-n / p} .
\end{aligned}
$$

Now, assume that $n=2$, then since $q>1$ and $\log (1+t) \preceq t^{1 / 2 q}$, for each $t \geq 1$, we obtain

$$
\frac{1}{(\delta(y))^{\lambda}} G(x, y) \preceq \frac{(\delta(x))^{1 / q-\lambda}}{|x-y|^{1 / q}} .
$$

So, by the Hölder inequality and Lemma 2.8 , it follows that

$$
\begin{aligned}
I_{1} & \preceq\|f\|_{p}(\delta(x))^{1 / q-\lambda}\left(\int_{D_{1}} \frac{1}{|x-y|} d y\right)^{1 / q} \\
& \preceq\|f\|_{p}(\delta(x))^{1 / q-\lambda}\left(\int_{0}^{((\sqrt{5}+1) / 2) \delta(x)} d r\right)^{1 / q} \\
& \preceq\|f\|_{p}(\delta(x))^{2 / q-\lambda}=\|f\|_{p}(\delta(x))^{2-\lambda-2 / p} .
\end{aligned}
$$

Next, by (1.1), we have for each $x \in D$ and $y \in D_{2}$

$$
\frac{1}{(\delta(y))^{\lambda}} G(x, y) \sim \frac{\delta(x)(\delta(y))^{1-\lambda}}{|x-y|^{n}} .
$$

Then, using the Hölder inequality and Lemma 2.8, we obtain

$$
I_{2} \preceq\|f\|_{p}\left(\int_{D_{2}}\left(\frac{\delta(x)(\delta(y))^{1-\lambda}}{|x-y|^{n}}\right)^{q} d y\right)^{1 / q}
$$

For each $y \in D_{2}$, it follows from Lemma 2.8 that $\delta(y) \preceq|x-y|$. So, we will discuss two cases.

Case 3. If $\lambda \leq 1$, it follows that

$$
\begin{aligned}
I_{2} & \preceq\|f\|_{p} \delta(x)\left(\int_{D_{2}} \frac{1}{|x-y|^{(n-1+\lambda) q}} d y\right)^{1 / q} \\
& \preceq\|f\|_{p} \delta(x)\left(\int_{((\sqrt{5}-1) / 2) \delta(x)}^{d} r^{n-1-(n-1+\lambda) q} d r\right)^{1 / q} .
\end{aligned}
$$


Thus, we distinguish the following two subcases.

(a) If $\lambda \leq 1-n / p$, then from (2.35) it follows that

$$
\begin{aligned}
I_{2} & \preceq\|f\|_{p} \delta(x)\left(\int_{((\sqrt{5}-1) / 2) \delta(x)}^{d} r^{(1-n-\lambda p) /(p-1)} d r\right)^{1 / q} \\
& \preceq\|f\|_{p} \delta(x) \begin{cases}\left(\log \frac{2 d}{\delta(x)}\right)^{1 / q} & \text { if } \lambda=1-\frac{n}{p} \\
1 & \text { if } \lambda<1-\frac{n}{p} .\end{cases}
\end{aligned}
$$

(b) If $1-n / p<\lambda \leq 1$, then by (2.34) we obtain

$$
\begin{aligned}
I_{2} & \preceq\|f\|_{p}(\delta(x))^{2-\lambda-n / p}\left(\int_{D_{2}} \frac{(\delta(x))^{(\lambda+n / p-1) q}}{|x-y|^{(n-1+\lambda) q}} d y\right)^{1 / q} \\
& =\|f\|_{p}(\delta(x))^{2-\lambda-n / p}\left(\int_{(((\sqrt{5}-1) / 2) \delta(x) \leq|x-y| \leq d)} \frac{1}{|x-y|^{n}} d y\right)^{1 / q} \\
& \preceq\|f\|_{p}(\delta(x))^{2-\lambda-n / p} .
\end{aligned}
$$

Case 4. If $\lambda>1$, then from (2.33) it follows that

$$
\begin{aligned}
I_{2} & \preceq\|f\|_{p}(\delta(x))^{2-\lambda-n / p}\left(\int_{D_{2}}\left(\frac{\delta(x)}{\delta(y)}\right)^{(\lambda-1) q} \frac{(\delta(x))^{n /(p-1)}}{|x-y|^{n+n / p-1}} d y\right)^{1 / q} \\
& \preceq\|f\|_{p}(\delta(x))^{2-\lambda-n / p}\left(\int_{D_{2}}\left(\frac{\delta(x)}{\delta(y)}\right)^{(\lambda-1) q} \frac{1}{|x-y|^{n}} d y\right)^{1 / q} .
\end{aligned}
$$

Since $(\lambda-1) q \in] 0,1[$, it follows from [ 8 , Corollary 2.8] that

$$
I_{2} \preceq\|f\|_{p}(\delta(x))^{2-\lambda-n / p} .
$$

This completes the proof.

Remark 2.9. By taking $p=+\infty$ (i.e., $q=1$ ), in Propositions 2.3 and 2.7, we find again the results of Mâagli in [8].

\section{First existence result}

In this section, we are interested in the existence of positive solutions for problem (1.5). We recall that $h_{0}$ is a fixed nontrivial nonnegative harmonic function in $D$, which is continuous in $\bar{D}$. Let $\varphi$ be a nontrivial nonnegative continuous function on $\partial D$.

We denote by $H_{D} \varphi$ the solution of the Dirichlet problem

$$
\Delta w=0 \quad \text { in } D, \quad w_{\mid \partial D}=\varphi .
$$

The main result of this section is the following. 
Theorem 3.1. Assume $\left(H_{1}\right)-\left(H_{2}\right)$. Then there exists a constant $c>1$ such that if $\varphi \geq c h_{0}$ on $\partial D$, then problem (1.5) has a positive continuous solution satisfying for each $x \in D$

$$
h_{0}(x) \leq u(x) \leq H_{D} \varphi(x)
$$

To prove Theorem 3.1, we need the following lemma.

For a fixed $q \in K^{+}(D)$, put

$$
\Gamma_{q}=\{v \in K(D):|v| \leq q\}
$$

then, we have

Lemma 3.2. Let $q$ be a nonnegative function belonging to $K(D)$, the family of functions

$$
\mathfrak{F}_{q}=\left\{\int_{D} G(\cdot, y) v(y) d y: v \in \Gamma_{q}\right\}
$$

is uniformly bounded and equicontinuous in $\bar{D}$, and consequently, it is relatively compact in $C_{0}(D)$.

Proof. Let $q \in K(D)$ and $T$ be the operator defined on $\mathfrak{F}_{q}$ by

$$
T v(x)=\int_{D} G(x, y) v(y) d y .
$$

By Proposition 2.1(i), we obtain

$$
\sup _{x \in D}|T v(x)| \leq \sup _{x \in D} \int_{D} G(x, y) q(y) d y<\infty .
$$

Then the family $T\left(\mathfrak{F}_{q}\right)$ is uniformly bounded.

Next, we propose to prove the equicontinuity of $T\left(\mathfrak{F}_{q}\right)$ in $\bar{D}$.

Let $v \in \mathfrak{F}_{q}, x_{0} \in D$, and $\alpha>0$. Let $x, x^{\prime} \in B\left(x_{0}, \alpha\right) \cap D$.

Then

$$
\left|T v(x)-T v\left(x^{\prime}\right)\right| \leq\left|V q(x)-V q\left(x^{\prime}\right)\right| .
$$

Since, by Proposition 2.1(i), $V q \in C_{0}(D)$, it follows that

$$
\left|T v(x)-T v\left(x^{\prime}\right)\right| \longrightarrow 0 \quad \text { as }\left|x-x^{\prime}\right| \longrightarrow 0 .
$$

Similarly, we have $\lim _{x \rightarrow \partial D} T v(x)=0$. Which implies that the family $T\left(\mathfrak{F}_{q}\right)$ is equicontinuous in $\bar{D}$.

Finally, by Ascoli's theorem, the family $T\left(\mathfrak{F}_{q}\right)$ is relatively compact in $C_{0}(D)$. Which completes the proof.

Proof of Theorem 3.1. We will use a fixed-point argument.

Let $c=1+\alpha_{\psi}$, where $\alpha_{\psi}$ is the constant defined by (2.2) associated to the function $\psi$ given in $\left(\mathrm{H}_{2}\right)$. Let $\varphi \in C^{+}(\partial D)$ such that $\varphi \geq c h_{0}$ on $\partial D$. 
We consider the set $\Lambda$ given by

$$
\Lambda=\left\{u \in C(\bar{D}): h_{0} \leq u \leq H_{D} \varphi\right\} .
$$

Since $\varphi \geq c h_{0}$ on $\partial D$, we obtain

$$
H_{D} \varphi \geq c h_{0} \text { on } D \text {. }
$$

So $\Lambda$ is a nonempty closed bounded and convex set in $C(\bar{D})$.

For each $u \in \Lambda$, define

$$
T u(x)=H_{D} \varphi(x)-\int_{D} G(x, y) f(y, u(y)) d y, \quad \forall x \in D .
$$

Now, we will prove that the family $T \Lambda$ is relatively compact in $C(\bar{D})$.

For each $y \in D$ and $u \in \Lambda$, we have by $\left(\mathrm{H}_{2}\right)$

$$
0 \leq f(y, u(y)) \leq \frac{\theta\left(y, h_{0}(y)\right)}{h_{0}(y)} h_{0}(y) \preceq c \psi(y) .
$$

with $c=\sup _{y \in D} h_{0}(y)$. Then, the function $y \rightarrow f(y, u(y)) \in \Gamma_{c \psi}$.

Hence the family

$$
\left\{\int_{D} G(\cdot, y) f(y, u(y)) d y: u \in \Lambda\right\} \subseteq \mathfrak{F}_{c \psi}
$$

So, using Lemma 3.2 and the fact that $H_{D} \varphi$ is continuous in $\bar{D}$, we conclude that $T \Lambda$ is a relatively compact set in $C(\bar{D})$.

Next, we intend to show that $T$ maps $\Lambda$ to itself.

It's obvious to see that

$$
T u(x) \leq H_{D} \varphi(x), \quad \forall x \in D .
$$

Moreover, from $\left(\mathrm{H}_{1}\right)$, and by using (3.11), (2.4), and (3.10), we obtain that for each $x \in D$

$$
T u(x) \geq H_{D} \varphi(x)-\alpha_{\psi} h_{0}(x) \geq h_{0}(x)
$$

which proves that $T \Lambda \subset \Lambda$.

Now, let us prove the continuity of the operator $T$ in $\Lambda$ in the supremum norm. Let $\left(u_{k}\right)_{k}$ be a sequence in $\Lambda$ which converges uniformly to a function $u$ in $\Lambda$. Then, for each $x \in D$, we have

$$
\left|T u_{k}(x)-T u(x)\right| \leq \int_{D} G(x, y)\left|f\left(y, u_{k}(y)\right)-f(y, u(y))\right| d y .
$$

On the other hand, by hypothesis $\left(\mathrm{H}_{1}\right)$, we have

$$
\left|f\left(y, u_{k}(y)\right)-f(y, u(y))\right| \leq 2 h_{0}(y) \psi(y) \preceq \psi(y) .
$$


Since $V \psi \in C_{0}(D)$, we conclude by the continuity of $f$ with respect to the second variable and the dominated convergence theorem that

$$
\forall x \in \bar{D}, \quad T u_{k}(x) \longrightarrow T u(x) \quad \text { as } k \longrightarrow+\infty
$$

Since $T \Lambda$ is a relatively compact family in $C(\bar{D})$, therefore the pointwise convergence implies the uniform convergence, namely,

$$
\left\|T u_{k}-T u\right\|_{\infty} \longrightarrow 0 \quad \text { as } k \longrightarrow+\infty .
$$

Thus, $T$ is a compact mapping on $\Lambda$.

Finally the Schauder fixed-point theorem implies the existence of $u \in \Lambda$ such that $T u=$ $u$, that is, for each $x \in D$

$$
u(x)=H_{D} \varphi(x)-\int_{D} G(x, y) f(y, u(y)) d y .
$$

Now, let us verify that $u$ is a solution of problem (1.5).

Since $\psi \in K(D)$, it follows from Proposition 2.1(ii), that $\psi \in L_{\mathrm{loc}}^{1}(D)$.

Furthermore, we have $f(\cdot, u) \leq c \psi$, then $f(\cdot, u) \in L_{\text {loc }}^{1}(D)$ and $V(f(\cdot, u)) \in \mathfrak{F}_{c \psi}$. So by Lemma 3.2, we have

$$
V(f(\cdot, u)) \in C_{0}(D) \subset L_{\mathrm{loc}}^{1}(D)
$$

Thus, applying $\Delta$ to both sides of (3.20) and using the fact that $\Delta(V f)=-f$, we obtain, that $u$ satisfies the elliptic differential equation

$$
\Delta u=f(\cdot, u) \quad \text { in } D \text { (in the sense of distributions). }
$$

Moreover, since $H_{D} \varphi=\varphi$ in $\partial D$ and $V(f(\cdot, u)) \in C_{0}(D)$, we conclude that $u_{\mid \partial D}=\varphi$. So $u$ is a positive continuous solution of problem (1.5).

Now, let us state another comparison result for the solution $u$ of problem (1.5), in the case of the special nonlinearity $f(x, t)=q(x) \Phi(t)$.

For this aim, suppose that the following hypotheses on $q$ and $\Phi$ are adopted.

(i) $\Phi:(0, \infty) \rightarrow(0, \infty)$ is continuously differentiable nonincreasing function.

(ii) $q$ is a nontrivial nonnegative function on $D$ such that

$$
q \in C_{\mathrm{loc}}^{\alpha}(D), 0<\alpha<1, \forall c>0, \quad x \longrightarrow \frac{q(x)}{\delta(x)} \Phi(c \delta(x)) \in K(D) .
$$

Moreover, let $F$ be the function defined on $[0, \infty)$ by

$$
F(t)=\int_{0}^{t} \frac{1}{\Phi(s)} d s
$$

It is obviously seen, from hypotheses adopted on $\Phi$, that the function $F$ is a bijection from $[0, \infty)$ to itself. Then, we have the following. 
Theorem 3.3. Let $u$ be the solution given by (3.20) of the following problem:

$$
\Delta u+q \Phi(u)=0, \quad \text { in } D, \quad u_{\mid \partial D}=\varphi .
$$

Then, we have $u \in C^{2+\alpha}(D) \cap C(\bar{D})$. Further, $u$ satisfies on $D$

$$
u(x) \leq \min \left(H_{D} \varphi(x), F^{-1}\left(H_{D}(F \circ \varphi)(x)-V q(x)\right)\right) .
$$

Proof. Let $v$ be the function defined on $D$ by

$$
v=F(u)-H_{D}(F \circ \varphi)+V q .
$$

Then $v \in C^{2}(D)$ and we have

$$
\Delta v=\frac{1}{\Phi(u)} \Delta u-\frac{\Phi^{\prime}(u)}{(\Phi(u))^{2}}|\nabla u|^{2}-q=-\frac{\Phi^{\prime}(u)}{(\Phi(u))^{2}}|\nabla u|^{2} .
$$

Thus, $\Delta v \geq 0$. In addition, since $V q \in C_{0}(D)$, it follows that $v \in C_{0}(D)$. Then, the maximum principle (see [6, pages 465-466]) implies that $v \leq 0$, in $D$. This completes the proof.

Remark 3.4. (1) Let $\lambda>0$ and $\varphi(x)=\lambda, \forall x \in \partial D$. Then, we have for each $x \in D$,

$$
H_{D}(F \circ \varphi)(x)-V q(x)=F(\lambda)(x)-V q(x) \leq F(\lambda) .
$$

Thus for each $x \in D$,

$$
F^{-1}\left(H_{D}(F \circ \varphi)(x)-V q(x)\right) \leq \lambda=H_{D} \varphi(x) .
$$

Therefore, from (3.26) we have for each $x \in D$,

$$
h_{0}(x) \leq u(x) \leq F^{-1}\left(H_{D}(F \circ \varphi)(x)-V q(x)\right) .
$$

(2) By hypothesis (i), we have

$$
\Phi\left(\|u\|_{\infty}\right) \geq \Phi\left(\|\varphi\|_{\infty}\right)
$$

Therefore,

$$
h_{0}(x) \leq u(x) \leq H_{D} \varphi(x)-\Phi\left(\|\varphi\|_{\infty}\right) V q(x)
$$

Then we have

$$
h_{0} \leq u \leq \min \left(H_{D} \varphi-\Phi\left(\|\varphi\|_{\infty}\right), F^{-1}\left(H_{D}(F \circ \varphi)-V q\right)\right) .
$$

Example 3.5. Let $h_{0}$ be a nontrivial nonnegative harmonic function, which is continuous on $\bar{D}$. Then, from [14], there exists $c_{1}$ such that for each $x \in D$

$$
h_{0}(x) \geq c_{1} \delta(x)
$$


Let $\alpha>0$, and $f$ be a nonnegative measurable function on $D \times(0, \infty)$, continuous with respect to the second variable satisfying

$$
f(x, t) \preceq t^{-\alpha}(\delta(x))^{\alpha+1} q(x),
$$

where the function $q$ belongs to $K^{+}(D)$.

Then, there exists $c>0$ such that if $\varphi \geq(1+c) h_{0}$ on $\partial D$, the problem

$$
\begin{gathered}
\Delta u=f(\cdot, u) \quad \text { (in the sense of distributions) } \\
u>0 \quad \text { in } D, \quad u_{\mid \partial D}=\varphi,
\end{gathered}
$$

has a positive continuous solution in $\bar{D}$ satisfying

$$
h_{0}(x) \leq u(x) \leq H_{D} \varphi(x) .
$$

\section{Second existence result}

In this section, we prove the following result for problem (1.8).

Theorem 4.1. Assume $\left(H_{3}\right)-\left(H_{4}\right)$. Then problem (1.8) has a positive solution $u \in C_{0}(D)$. Moreover there exist positive constants $a$ and $b$, such that

$$
a \delta(x) \leq u(x) \leq b
$$

Proof. By $\left(\mathrm{A}_{2}\right)$ and $\left(\mathrm{H}_{4}\right)$, the function $q \in K^{+}(D)$. Then, from Proposition 2.1(i), we have $V q \in C_{0}(D)$. So, $M:=\sup _{x \in D}(V q(x))<\infty$.

From $\left(\mathrm{A}_{4}\right)$, there exists $b>0$ such that $M k(b) \leq b$.

On the other hand, by $\left(\mathrm{A}_{1}\right)$, there exists a compact $K \subset D$ such that

$$
0<\int_{K} \delta(y) p(y) d y<\infty .
$$

Furthermore, by (1.1), there exists $\alpha>0$ such that for each $x, y$ in $D$

$$
G(x, y) \geq \alpha \delta(x) \delta(y) .
$$

Next, let $r$ be the constant given by

$$
r:=\inf _{y \in K} \delta(y)
$$

Then, from $\left(\mathrm{H}_{4}\right)$, there exists $a>0$ such that

$$
\alpha h(a r) \int_{K} \delta(y) p(y) d y \geq a .
$$

Now, let $\Omega$ be the convex set

$$
\Omega:=\left\{u \in C_{0}(D): a \delta(x) \leq u(x) \leq b\right\}
$$


and $S$ be the operator defined on $\Omega$ by

$$
\operatorname{Su}(x)=\int_{D} G(x, y) \rho(y, u(y)) d y .
$$

We will prove that $S$ is a compact mapping on $\Omega$.

By $\left(\mathrm{H}_{4}\right)$, we have for each $u \in \Omega$

$$
\rho(\cdot, u) \leq k(b) q=\tilde{q} .
$$

Since $q \in K^{+}(D)$, it follows that the function $y \rightarrow \rho(y, u(y)) \in \Gamma_{\tilde{q}}$.

Hence, the family

$$
\left\{\int_{\Omega} G(\cdot, y) \rho(y, u(y)) d y: u \in \Omega\right\} \subseteq \mathfrak{F}_{\tilde{q}} .
$$

Consequently, by Lemma 3.2, the family $S(\Omega)$ is relatively compact in $C_{0}(D)$. Next, we need to verify that for $u \in \Omega$ and $x \in D$, we have

$$
a \delta(x) \leq S u(x) \leq b
$$

Let $u \in \Omega$ and $x \in D$, then by $\left(\mathrm{H}_{4}\right)$, we have

$$
\begin{aligned}
S u(x) & \leq \int_{D} G(x, y) q(y) k(u(y)) d y \\
& \leq k(b) \int_{D} G(x, y) q(y) d y \\
& \leq M k(b) \leq b .
\end{aligned}
$$

On the other hand, from $\left(\mathrm{H}_{4}\right)$ and using (1.1) and (4.5), we have

$$
\begin{aligned}
S u(x) & \geq \alpha \delta(x) \int_{D} \delta(y) p(y) h(u(y)) d y \\
& \geq \alpha \delta(x) \int_{K} \delta(y) p(y) h(a \delta(y)) d y \\
& \geq \delta(x)\left[\alpha h(a r) \int_{K} \delta(y) p(y) d y\right] \geq a \delta(x) .
\end{aligned}
$$

Thus, it follows that $S(\Omega) \subset \Omega$.

Now, we consider a sequence $\left(u_{k}\right)_{k}$ in $\Omega$ which converges uniformly to $u$ in $\Omega$. Since $\rho$ is continuous with respect to the second variable, we deduce by the dominated convergence theorem that for all $x \in D$,

$$
S u_{k}(x) \longrightarrow \operatorname{Su}(x) \text { as } k \longrightarrow+\infty \text {. }
$$

Therefore, using the fact that $S(\Omega)$ is relatively compact in $C_{0}(D)$, we conclude that $\left\|S u_{k}-S u\right\|_{\infty}$ as $k \rightarrow+\infty$. Hence $S$ is a compact mapping from $\Omega$ to itself. Then by the 
Schauder fixed-point theorem, there exists a function $u \in \Omega$ such that

$$
u(x)=\int_{D} G(x, y) \rho(y, u(y)) d y .
$$

Now, since $q \in K^{+}(D)$ then by Proposition 2.1(ii), we have $\rho(\cdot, u) \in L_{\text {loc }}^{1}(D)$ and $V(\rho(\cdot$, $u)) \in C_{0}(D) \subset L_{\text {loc }}^{1}(D)$.

So, $u$ satisfies (in the sense of distributions) $\Delta u=-\rho(\cdot, u)$ in $D$. Moreover, $\lim _{x \rightarrow \partial D} u(x)$ $=\lim _{x \rightarrow \partial D} V(\rho(\cdot, u(\cdot)))(x)=0$. So $u$ is a solution of problem $(1.8)$.

Example 4.2. Let $p>n / 2$ and $f \in L_{+}^{p}(D)$. Assume that the function $g:(0, \infty) \rightarrow[0, \infty)$ is a nontrivial continuous and nondecreasing function satisfying

$$
\lim _{t \rightarrow 0^{+}} \frac{g(t)}{t}=+\infty, \quad \lim _{t \rightarrow+\infty} \frac{g(t)}{t}=0
$$

Then for each $\lambda<2-n / p$ the problem

$$
\Delta u=-(\delta(x))^{-\lambda} f(x) g(u) \quad \text { in } D, \quad u_{\mid \partial D}=0,
$$

has a positive solution $u \in C_{0}(D)$. Moreover, from Proposition 2.7, we have for each $x \in$ $D$,

$$
u(x) \leq \begin{cases}c\|f\|_{p}(\delta(x))^{2-n / p-\lambda}, & \text { if } 1-\frac{n}{p}<\lambda<2-\frac{n}{p}, \\ c\|f\|_{p} \delta(x)\left(\log \frac{2 d}{\delta(x)}\right)^{(p-1) / p}, & \text { if } \lambda=1-\frac{n}{p} \\ c\|f\|_{p} \delta(x), & \text { if } \lambda<1-\frac{n}{p} .\end{cases}
$$

\section{Acknowledgments}

I would like to sincerely thank my doctoral thesis advisor Professor Habib Maâgli for his support during the preparation of this work. Thanks also go to the referee for the careful review of the paper.

\section{References}

[1] M. Aizenman and B. Simon, Brownian motion and Harnack inequality for Schrödinger operators, Communications on Pure and Applied Mathematics 35 (1982), no. 2, 209-273.

[2] S. Athreya, On a singular semilinear elliptic boundary value problem and the boundary Harnack principle, Potential Analysis. An International Journal Devoted to the Interactions between Potential Theory, Probability Theory, Geometry and Functional Analysis 17 (2002), no. 3, 293 301.

[3] H. Brezis and S. Kamin, Sublinear elliptic equations in $\mathbf{R}^{n}$, Manuscripta Mathematica 74 (1992), no. $1,87-106$.

[4] K. L. Chung and Z. X. Zhao, From Brownian Motion to Schrödinger's Equation, Grundlehren der Mathematischen Wissenschaften, vol. 312, Springer, Berlin, 1995. 
[5] R. Dalmasso, Existence and uniqueness results for polyharmonic equations, Nonlinear Analysis. Theory, Methods \& Applications. An International Multidisciplinary Journal. Series A: Theory and Methods 36 (1999), no. 1, 131-137.

[6] R. Dautray and J.-L. Lions, Analyse mathématique et calcul numérique pour les sciences et les techniques. Vol. 1, L'oprateur de Laplace, INSTN: Collection Enseignement. [INSTN: Teaching Collection], Masson, Paris, 1987.

[7] N. J. Kalton and I. E. Verbitsky, Nonlinear equations and weighted norm inequalities, Transactions of the American Mathematical Society 351 (1999), no. 9, 3441-3497.

[8] H. Mâagli, Inequalities for the Riesz potentials, Archives of Inequalities and Applications. An International Journal for Theory and Applications 1 (2003), no. 3-4, 285-294.

[9] H. Mâagli and L. Mâatoug, Singular solutions of a nonlinear equation in bounded domains of $\mathbf{R}^{2}$, Journal of Mathematical Analysis and Applications 270 (2002), no. 1, 230-246.

[10] H. Mâagli and M. Zribi, On a new Kato class and singular solutions of a nonlinear elliptic equation in bounded domains of $\mathbb{R}^{n}$, to appear in Positivity.

[11] S. C. Port and C. J. Stone, Brownian motion and classical potential theory, Probability and Mathematical Statistics, Academic Press, New York, 1978.

[12] M. Selmi, Inequalities for Green functions in a Dini-Jordan domain in $\mathbf{R}^{2}$, Potential Analysis. An International Journal Devoted to the Interactions between Potential Theory, Probability Theory, Geometry and Functional Analysis 13 (2000), no. 1, 81-102.

[13] N. Zeddini, Positive solutions for a singular nonlinear problem on a bounded domain in $\mathbb{R}^{2}$, Potential Analysis. An International Journal Devoted to the Interactions between Potential Theory, Probability Theory, Geometry and Functional Analysis 18 (2003), no. 2, 97-118.

[14] Z. X. Zhao, Uniform boundedness of conditional gauge and Schrödinger equations, Communications in Mathematical Physics 93 (1984), no. 1, 19-31.

Faten Toumi: Département de Mathématiques, Faculté des Sciences de Tunis, Campus Universitaire, 2092 Tunis, Tunisia

E-mail address: faten.toumi@fsb.rnu.tn 


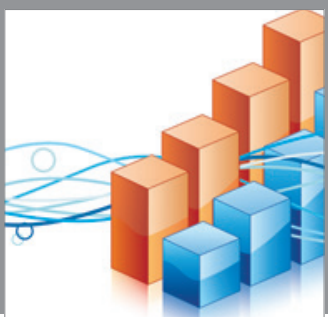

Advances in

Operations Research

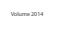

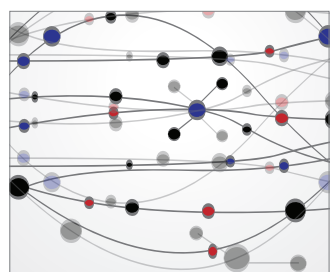

\section{The Scientific} World Journal
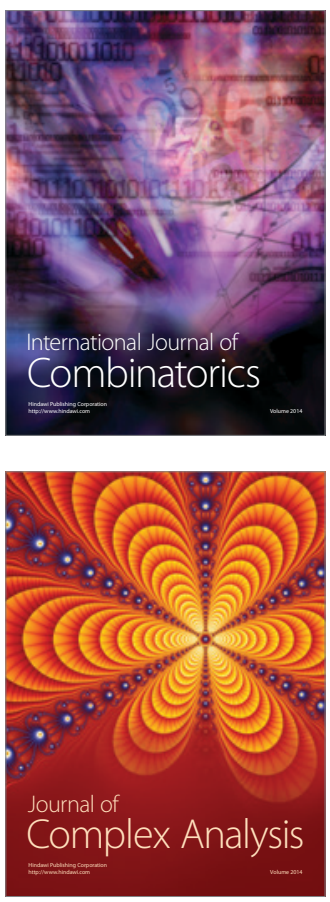

International Journal of

Mathematics and

Mathematical

Sciences
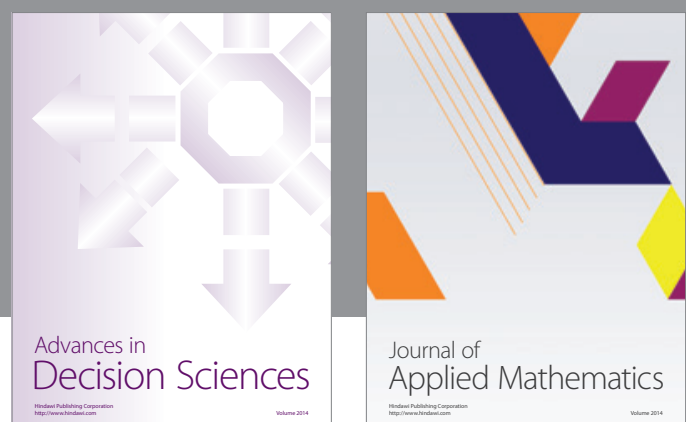

Journal of

Applied Mathematics
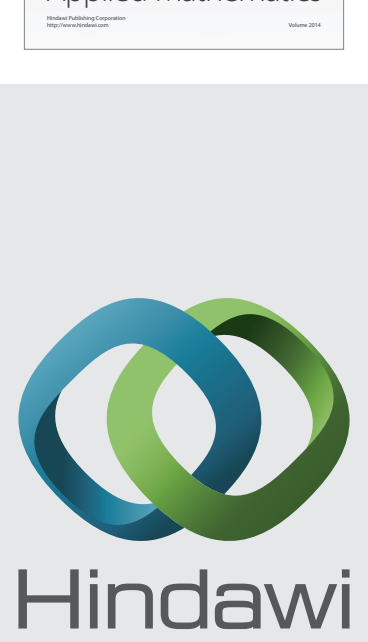

Submit your manuscripts at http://www.hindawi.com
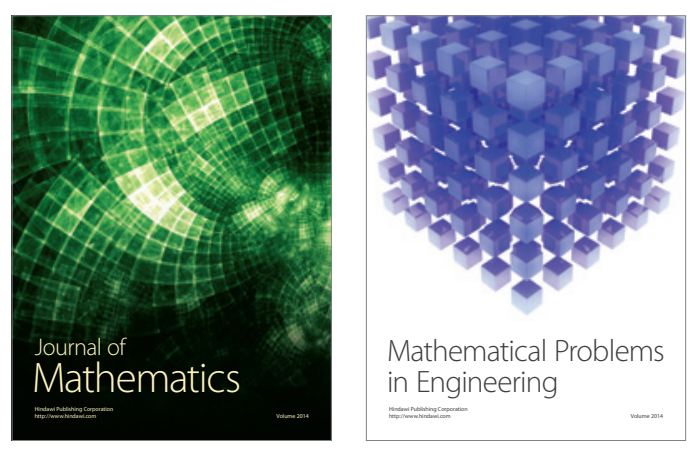

Mathematical Problems in Engineering
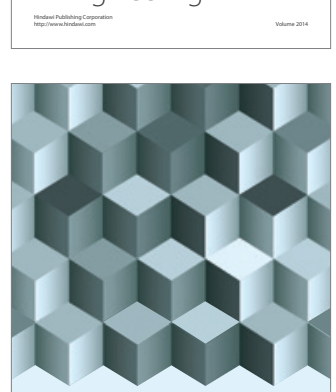

Journal of

Function Spaces
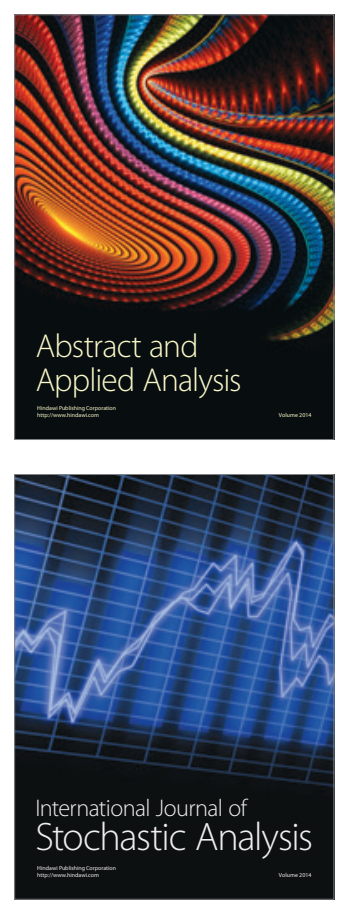

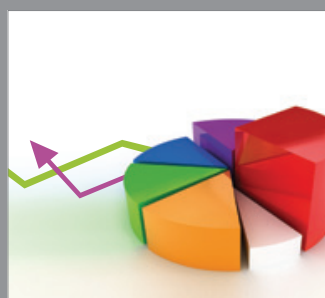

ournal of

Probability and Statistics

Promensencen
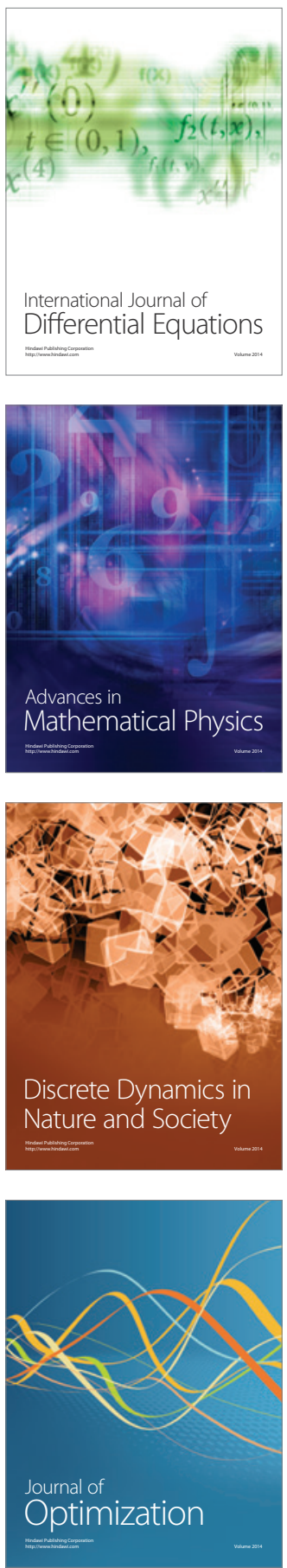